\title{
ON THE ICOSAHEDRON INEQUALITY OF LÁSZLÓ FEJES-TÓTH
}

\author{
ÁKOS G. HORVÁTH
}

\begin{abstract}
In this paper we deal with the problem of finding the maximal volume polyhedra with a prescribed property and inscribed in the unit sphere. We generalize an inequality (called icosahedron inequality) of L. Fejes-Tóth which has the following interesting consequence: the regular icosahedron has maximal volume in the class of the polyhedra having twelve vertices and inscribed in the unit sphere. We give an upper bound for the volume of such star-shaped (with respect to the origin) simplicial polyhedra, whose number of faces, and also the list of the maximal edge lengths of the faces are given. As a consequence of this inequality we prove a conjecture which states that the maximal volume polyhedron spanned by the vertices of two regular simplices with common centroid is the cube.
\end{abstract}

Mathematics subject classification (2010): 52A40, 52A38, $26 \mathrm{~B} 15$.

Keywords and phrases: Convex hull, volume inequality.

\section{REFERENCES}

[1] J. D. Berman AND K. HANES, Volumes of polyhedra inscribed in the unit sphere in $E^{3}$, Math. Ann. 188 (1970), 78-84.

[2] P. Brass, W. Moser And J. PACh, Research Problems in Discrete Geometry, Springer, New York, 2005.

[3] H. T. Croft, K. J. Falconer and R. K. Guy, Unsolved Problems in Geometry, Vol. 2, Springer, New York, 1991.

[4] J. CASEY, A treatise on spherical trigonometry, and its application to Geodesy and Astronomy, with numerous examples, Hodges, Figgis and CO., Grafton-ST. London: Longmans, Green, and CO., 1889.

[5] L. Fejes-Tóth, Regular Figures, The Macmillan Company, New York, 1964.

[6] Á. G. HoRváth, Maximal convex hull of connecting simplices, Studies of the University of Zilina 22/1 (2008), 7-19.

[7] Á. G. HoRvÁth, On an extremal problem connected with simplices, Beiträge zur Geometrie und Algebra (2013), doi:10.1007/s13366-013-0151-9.

[8] Á. G. Horváth, Computations with Mathematica 10, an addendum to the paper "On the icosahedron inequality of László Fejes-Tóth", DOI: 10.13140/2.1.3416.6566 (2014) or http://www.math.bme.hu/_ghorvath/calculationmath.pdf.

[9] Á. G. Horváth, Zs. LÁngI, Maximum volume polytopes inscribed in the unit sphere, (2014), http://arxiv.org/abs/1402.6496.

[10] N. MUtoH, The polyhedra of maximal volume inscribed in the unit sphere and of minimal volume circumscribed about the unit sphere, JCDCG, Lecture Notes in Computer Science 2866 (2002), 204214. 